\title{
AN INITIAL STABILITY OF PLATES IN VARIOUS CONSERVATIVE LOAD CONDITIONS BY THE BOUNDARY ELEMENT METHOD
}

\author{
Michal Guminiak \\ Institute of Structural Engineering, Poznan University of Technology \\ Poznan, Poland \\ michal.guminiak@put.poznan.pl
}

\begin{abstract}
An initial stability of Kirchhoff plates by the Boundary Element Method (BEM) is presented in the paper. A plate is subjected by external in-plane normal and tangential conservative loadings acting in two perpendicular directions. The Betti's theorem is used to derive the boundary-domain integral equations. The direct version of the Boundary Element Method is presented with combination to simplified boundary conditions. The singular and non-singular approach of the boundary integrals derivation is used.
\end{abstract}

Keywords: initial stability of plates, the Boundary Element Method, fundamental solutions

\section{Introduction}

The Boundary Element Method (BEM) is often used in the theory of thin and thick plates. There is a number of contributions devoted to the application of the BEM to the stability analysis of plates. Shi [1] applied the BEM formulation for vibration and the initial stability problem of orthotropic thin plates and used the Bèzine technique [2] to establish the vector of plate curvatures inside a plate domain. Nerantzaki and Katsikadelis [3] solved the buckling problem of a plate with variable thickness. The authors applied the Analog Equation Method (AEM) connected to pure BEM [4]. A similar approach was applied by Chinnaboon, Chucheepsakul and Katsikadelis [5] to solve buckling analysis of plates. Katsikadelis and Babouskos [6] applied AEM in combination with the BEM to describe and solve the nonlinear flutter instability problem of thin dumped plates. In order to simplify the assembly of a set of algebraic equations and calculation procedures, Guminiak, Sygulski [7] and Guminiak [8] proposed a modified, simplified formulation of the boundary integral equations for a thin plate. Katsikadelis [9] solved the plate stability problem considering complex external in-plane loading condition. The author used a combination of the AEM-BEM approach and used difference operators to define the vector of curvatures inside a plate domain. In the present paper, an analysis of plate initial stability by the BEM will be presented. The 
analysis will focus on the modified formulation $[7,8]$ of thin plate bending. The Bèzine technique [2] will be established to directly derive the boundary-domain integral equations.

\section{Integral formulation of a thin plate stability problem}

The differential equation governing of plate initial stability has the form [10]:

$$
D \cdot \nabla^{4} w=-\widetilde{p}
$$

where $\widetilde{p}$ is the in-plane external load defined as

$$
\widetilde{p}=N_{x} \cdot \frac{\partial^{2} w}{\partial x^{2}}+2 N_{x y} \cdot \frac{\partial^{2} w}{\partial x \partial y}+N_{y} \cdot \frac{\partial^{2} w}{\partial y^{2}}
$$

On the plate boundary, the following variables are considered: shear force $\widetilde{T}_{n}$, bending moment $M_{n}$ and deflection $w$, angle of rotation in normal direction $\varphi_{n}$ and angle of rotation in tangent direction $\varphi_{s}$. The expression $\widetilde{T}_{n}(\mathbf{y})=T_{n}(\mathbf{y})+R_{n}(\mathbf{y})$ denotes shear force for clamped and simply-supported edges, wherein $\widetilde{T}_{n}(\mathbf{y})=V_{n}(\mathbf{y})$ on the boundary far from the corner and $\widetilde{T}_{n}(\mathbf{y})=R_{n}(\mathbf{y})$ on a small fragment of the boundary close to the corner [8]. The relation between $\varphi_{s}(\mathbf{y})$ and the deflection is known: $\varphi_{s}(\mathbf{y})=d w(\mathbf{y}) / d s$ and it can be evaluated using a finite difference scheme of the deflection with two or more adjacent nodal values. In this analysis, the employed finite difference scheme includes the deflections of two adjacent nodes. The boundary-domain integral equations are derived using the Betti's theorem and they have the form:

$$
\begin{aligned}
& c(\mathbf{x}) \cdot w(\mathbf{x})+\int_{\Gamma}\left[T_{n}^{*}(\mathbf{y}, \mathbf{x}) \cdot w(\mathbf{y})-M_{n s}^{*}(\mathbf{y}, \mathbf{x}) \cdot \frac{d w(\mathbf{y})}{d s}-M_{n}^{*}(\mathbf{y}, \mathbf{x}) \cdot \varphi_{n}(\mathbf{y})\right] \cdot d \Gamma(\mathbf{y})= \\
& =\int_{\Gamma}\left[\widetilde{T}_{n}(\mathbf{y}) \cdot w^{*}(\mathbf{y}, \mathbf{x})-M_{n}(\mathbf{y}) \cdot \varphi_{n}^{*}(\mathbf{y}, \mathbf{x})\right] \cdot d \Gamma(\mathbf{y})+\int_{\Omega} \widetilde{p} \cdot w^{*}(\mathbf{y}, \mathbf{x}) \cdot d \Omega(\mathbf{y}) \\
& c(\mathbf{x}) \cdot \varphi_{n}(\mathbf{x})+\int_{\Gamma}\left[\bar{T}_{n}^{*}(\mathbf{y}, \mathbf{x}) \cdot w(\mathbf{y})-\bar{M}_{n s}^{*}(\mathbf{y}, \mathbf{x}) \cdot \frac{d w(\mathbf{y})}{d s}-\bar{M}_{n}^{*}(\mathbf{y}, \mathbf{x}) \cdot \varphi_{n}(\mathbf{y})\right] \cdot d \Gamma(\mathbf{y})= \\
& =\int_{\Gamma}\left[\widetilde{T}_{n}(\mathbf{y}) \cdot \bar{w}^{*}(\mathbf{y}, \mathbf{x})-M_{n}(\mathbf{y}) \cdot \bar{\varphi}_{n}^{*}(\mathbf{y}, \mathbf{x})\right] \cdot d \Gamma(\mathbf{y})+\int_{\Omega} \widetilde{p} \cdot \bar{w}^{*}(\mathbf{y}, \mathbf{x}) \cdot d \Omega(\mathbf{y})
\end{aligned}
$$

where the fundamental solution of biharmonic equation $\nabla^{4} w=(1 / D) \cdot \delta(\mathbf{y}-\mathbf{x})$ is given as a Green function 


$$
w^{*}(\mathbf{y}, \mathbf{x})=\frac{1}{8 \pi D} \cdot r^{2} \cdot \ln r
$$

for a thin isotropic plate, $\quad r=|\mathbf{y}-\mathbf{x}|, \quad \delta$ is the Dirac delta and $D=\left(E h_{\mathrm{p}}^{3}\right) /\left(12\left(1-v_{\mathrm{p}}^{2}\right)\right)$ is the plate stiffness. The coefficient $c(\mathbf{x})$ depends on the localization of point $\mathbf{x}$ and $c(\mathbf{x})=1$, when $\mathbf{x}$ is located inside the plate region, $c(\mathbf{x})=0.5$, when $\mathbf{x}$ is located on the smooth boundary and $c(\mathbf{x})=0$, when $\mathbf{x}$ is located outside the plate region. The second boundary-domain integral equation (4) can be derived by substituting a unit concentrated force $P^{*}=1$ by unit concentrated moment $M_{n}^{*}=1$. It is equivalent to differentiate the first boundary integral equation (3) on $n$ direction in point $\mathbf{x}$ on a plate boundary, wherein

$$
\begin{aligned}
& \left\{\bar{T}_{n}^{*}(\mathbf{y}, \mathbf{x}), \bar{M}_{n}^{*}(\mathbf{y}, \mathbf{x}), \bar{M}_{n s}^{*}(\mathbf{y}, \mathbf{x}), \bar{w}^{*}(\mathbf{y}, \mathbf{x}), \bar{\varphi}_{n}^{*}(\mathbf{y}, \mathbf{x}), \bar{\varphi}_{s}^{*}(\mathbf{y}, \mathbf{x})\right\}= \\
& =\frac{\partial}{\partial n(\mathbf{x})}\left\{T_{n}^{*}(\mathbf{y}, \mathbf{x}), M_{n}^{*}(\mathbf{y}, \mathbf{x}), M_{n s}^{*}(\mathbf{y}, \mathbf{x}), w^{*}(\mathbf{y}, \mathbf{x}), \varphi_{n}^{*}(\mathbf{y}, \mathbf{x}), \varphi_{s}^{*}(\mathbf{y}, \mathbf{x})\right\}
\end{aligned}
$$

\section{Assembly of a set of algebraic equations}

A plate is subjected by in-plane external loading (Fig. 1). The distribution of external loading along a plate edge is constant.

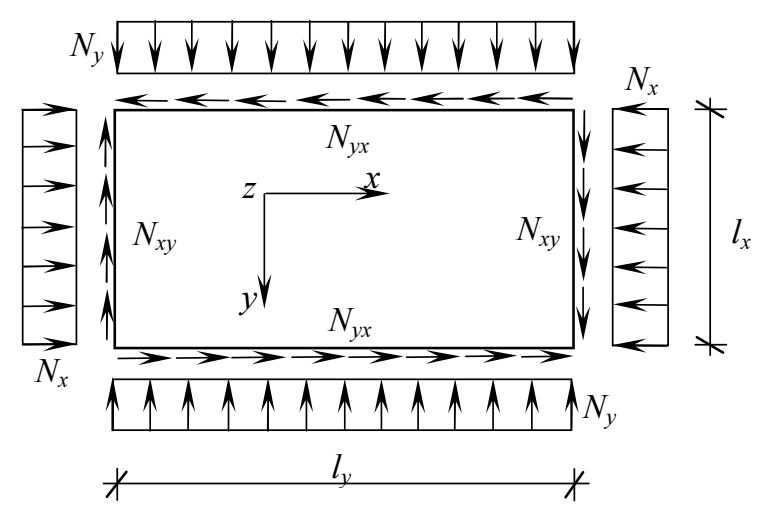

Fig. 1. A plate subjected by in-plane external loading

Let it be assumed that a plate boundary is discretized using elements of the constant type and a plate domain is divided into rectangular sub-domains. It is assumed that the relation between external in-plane forces $N_{x}, N_{y}$ and $N_{x y}$ is known, so that the problem is defined as a single-parameter issue. The set of algebraic equations has following form: 


$$
\left[\begin{array}{ccc}
\mathbf{G}_{\mathbf{B B}} & \mathbf{G}_{\mathbf{B s}} & -\lambda \cdot \mathbf{G}_{\mathbf{B} \widetilde{\mathbf{\kappa}}} \\
\boldsymbol{\Delta} & -\mathbf{I} & \mathbf{0} \\
\mathbf{G}_{\widetilde{\mathbf{\kappa}} \mathbf{B}} & \mathbf{G}_{\widetilde{\mathbf{\kappa}} \mathbf{s}} & -\lambda \cdot \mathbf{G}_{\widetilde{\mathbf{\kappa}} \tilde{\mathbf{K}}}+\mathbf{I}
\end{array}\right] \cdot\left\{\begin{array}{c}
\mathbf{B} \\
\varphi_{\mathbf{s}} \\
\widetilde{\mathbf{K}}
\end{array}\right\}=\left\{\begin{array}{l}
\mathbf{0} \\
\mathbf{0} \\
\mathbf{0}
\end{array}\right\}
$$

where the critical force $N_{\text {cr }}$ is expressed by eigenvalue multiplier $\lambda,\left(\lambda=N_{\text {cr }}\right)$. All of the designations appearing in matrix equation (6) are shown in Figure 2.

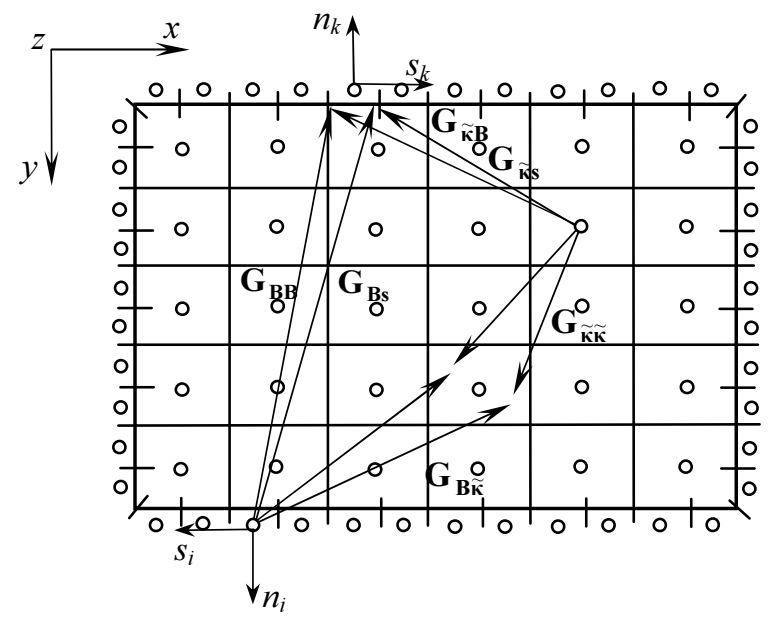

Fig. 2. Construction of the characteristic matrix

The boundary-domain integral equations are formulated in a singular and a nonsingular approach [8]. Integration of suitable fundamental functions is done in a local coordinate system $n_{i}, s_{i}$ connected with $i^{\text {th }}$ boundary element and next, these integrals must be transformed to $n_{k}, s_{k}$ coordinate system, connected with $k^{\text {th }}$ element. For a non-singular approach, the localization of a collocation point is defined by the parameter $\widetilde{\delta}$ which determines the distance from a plate edge or by nondimensional parameter $\varepsilon=\widetilde{\delta} / d$ where $d$ is the element length [7, 8]. The vector of unknowns consist: $\mathbf{B}$ - the vector of boundary independent variables, $\varphi_{\mathbf{s}}$ - the vector of additional parameters of the angle of rotation in the tangential direction, which depends on the boundary deflection in case of the free edge and $\widetilde{\mathbf{\kappa}}$ - vector specifying generalized curvatures inside a plate domain. The matrix $\mathbf{G}_{\mathbf{B B}}$ groups boundary integrals dependent on type of boundary and the matrix $\mathbf{G}_{\mathrm{Bs}}$ groups boundary integrals of functions $M_{n s}^{*}$ and $\bar{M}_{n s}^{*}$ in case of free edge occurrence and it is the additional matrix grouping boundary integrals corresponding with rotation in tangential direction $\varphi_{\mathbf{s}}$. The matrix $\mathbf{G}_{\mathbf{B} \widetilde{\kappa}}$ groups values of fundamental functions $w^{*}$ and $\bar{w}^{*}$ established in internal collocation points associated with rectangular inter- 
nal sub-surfaces. The matrix $\Delta$ groups the finite difference expressions for the angle of rotation in the tangential direction $\varphi_{\mathbf{s}}$ in terms of deflections at suitable, adjacent nodes and $\mathbf{I}$ is the unit matrix. In the computer program, deflections at two neighbouring nodes are used. Hence, for a clamped edge, a simply-supported edge and a free edge, two independent unknowns are always considered. Let the operator

$$
\alpha \cdot \frac{\partial^{2}}{\partial x^{2}}+2 \beta \cdot \frac{\partial^{2}}{\partial x \partial y}+\gamma \cdot \frac{\partial^{2}}{\partial y^{2}}
$$

acts on the first boundary-domain integral equation (3) wherein coefficients $\alpha, \beta, \gamma \in C$ are known. Matrices $\mathbf{G}_{\tilde{\mathbf{\kappa}} \mathbf{B}}, \mathbf{G}_{\widetilde{\mathbf{\kappa s}}}$ and $\mathbf{G}_{\widetilde{\mathbf{\kappa}} \tilde{\mathbf{\kappa}}}$ group boundary and domain integrals using fundamental functions obtained for the first boundary-domain integral equation (3) which was subjected by the operator (7). Elimination of boundary variables $\mathbf{B}$ and $\varphi_{\mathbf{s}}$ from matrix equation (6) leads to a standard eigenvalue problem

$$
\{\mathbf{A}-\tilde{\lambda} \cdot \mathbf{I}\} \cdot \widetilde{\mathbf{\kappa}}=\mathbf{0}
$$

where $\tilde{\lambda}=1 / \lambda$ and

$$
\mathbf{A}=\left\{\mathbf{G}_{\widetilde{\mathbf{\kappa}} \widetilde{\mathbf{K}}}-\left(\mathbf{G}_{\widetilde{\mathbf{K}} \mathbf{B}}-\mathbf{G}_{\widetilde{\mathbf{\kappa}} \mathbf{s}} \cdot \Delta\right) \cdot\left[\mathbf{G}_{\mathbf{B B}}+\mathbf{G}_{\mathbf{B s}}\right]^{-1} \cdot \mathbf{G}_{\mathbf{B} \tilde{\mathbf{K}}}\right\}
$$

\section{Modes of buckling}

The elements of the eigenvector $\widetilde{\mathbf{\kappa}}$ obtained after solution of the standard eigenvalue problem (8) present the plate curvatures. The set of the algebraic equations indispensable to calculate the elements of eigenvector $\mathbf{w}$ has the form

$$
\left[\begin{array}{ccc}
\mathbf{G}_{\mathbf{B B}} & \mathbf{G}_{\mathbf{B s}} & \mathbf{0} \\
\boldsymbol{\Delta} & -\mathbf{I} & \mathbf{0} \\
\mathbf{G}_{\mathbf{w B}} & \mathbf{G}_{\mathbf{w s}} & \mathbf{I}
\end{array}\right] \cdot\left\{\begin{array}{l}
\mathbf{B} \\
\varphi_{\mathbf{s}} \\
\mathbf{w}
\end{array}\right\}=\left\{\begin{array}{c}
\lambda \cdot \mathbf{G}_{\mathbf{B w}} \cdot \tilde{\mathbf{K}} \\
\mathbf{0} \\
\lambda \cdot \mathbf{G}_{\mathbf{w w}} \cdot \widetilde{\mathbf{K}}
\end{array}\right\}
$$

where the first and second equations $(10)_{1}$ and $(10)_{2}$ are obtained from the first and second equations of $(6)$ and the third equation $(10)_{3}$ is gotten by construction of the boundary integral equations for calculating the plate deflection in internal collocation points. The wanted displacement vector $\mathbf{w}$ can be calculated directly by elimination of boundary variables $\mathbf{B}$ and $\varphi_{\mathbf{s}}$

$$
\mathbf{w}=\lambda \cdot\left[\mathbf{G}_{\mathbf{w w}}-\left(\mathbf{G}_{\mathbf{w B}}-\mathbf{G}_{\mathbf{w s}} \cdot \boldsymbol{\Delta}\right) \cdot\left[\mathbf{G}_{\mathrm{BB}}+\mathbf{G}_{\mathrm{Bs}}\right]^{-1} \cdot \mathbf{G}_{\mathrm{Bw}}\right] \cdot \widetilde{\mathbf{K}}
$$




\section{Numerical examples}

The initial stability problem of rectangular plates with various boundary and load conditions is considered. The loaded plate edge must be supported. The critical value of the external loading is investigated. Each of the plate edges is divided by the boundary elements of the constant type with the same length. The set of the internal collocation points associated with sub-domains is regular. The plate properties are: Young modulus $E=205 \mathrm{GPa}$, Poisson ratio $v=0.3$. The following notations are assumed: BEM I - singular formulation of governing boundary-domain integral equations (3) and (4); BEM II - non-singular formulation of governing boundary-domain integral equations (3) and (4), the collocation point of single boundary element is located outside, near the plate edge, $\varepsilon=\tilde{\delta} / d=0.001[7,8]$. The critical force $N_{\mathrm{cr}}$ is expressed using non-dimensional term:

$$
\widetilde{N}_{\mathrm{cr}}=\frac{N_{\mathrm{cr}}}{D} \cdot l_{x} \cdot l_{y}
$$

where $D$ is the plate stiffness, $l_{x}$ and $l_{y}$ are the plate in-plane dimensions.

\subsection{Example 1}

A square plate, simply-supported on a whole edge and subjected by $N_{x}$ and $N_{y}$ in-plane forces is considered. The intensities of external in-plane forces are constant. The plate edges were divided into 64 boundary elements and the number of internal square sub-domains is equal to 256 . The results of the calculation are presented in Table 1 .

Table 1

Critical forces

\begin{tabular}{|c|c|c|c|c|c|c|}
\hline \multirow{2}{*}{$\tilde{N}_{\text {cr }}$} & \multicolumn{6}{|c|}{$\tilde{N}_{y} / \tilde{N}_{x},\left(\tilde{N}_{x}>0\right)$} \\
\cline { 2 - 7 } & -0.25 & 0.0 & 0.25 & 0.5 & 0.75 & 1.0 \\
\hline BEM I & 52.826 & 39.620 & 31.696 & 26.424 & 22.640 & 19.810 \\
\hline BEM II & 52.848 & 39.636 & 31.583 & 26.319 & 22.559 & 19.739 \\
\hline Analytical [10] & 52.638 & 39.478 & 31.708 & 26.424 & 22.649 & 19.818 \\
\hline
\end{tabular}

The first buckling mode for $N_{x}=N_{y}$ is shown in Figure 3 . 


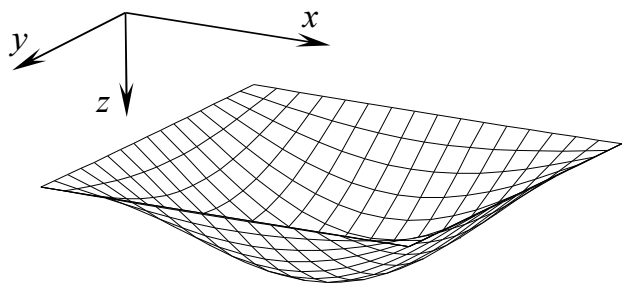

Fig. 3. The first buckling mode of the square plate, simply-supported on a whole edge for $N_{x}=N_{y}$

\subsection{Example 2}

A square plate clamped on a whole edge and subjected by $N_{x}$ and $N_{y}$ in-plane compressive forces is considered. The intensities of external in-plane forces are constant. The plate boundary and domain discretizations are the same as in the Example 1. The results of the calculation for $N_{x}=N_{y}$ are presented in Table 2.

Table 2

\section{Critical forces}

\begin{tabular}{|c|c|c|}
\hline \multicolumn{3}{|c|}{$\tilde{N}_{\text {cr }}$} \\
\hline BEM I & BEM II & Analytical [10] \\
\hline 52.784 & 52.784 & 52.605 \\
\hline
\end{tabular}

The first buckling mode for $N_{x}=N_{y}$ is shown in Figure 4 .

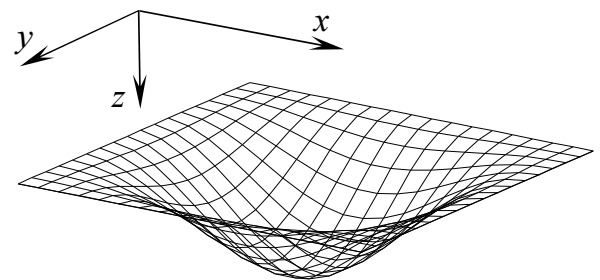

Fig. 4. The first buckling mode of the square plate, clamped on a whole edge for $N_{x}=N_{y}$

\subsection{Example 3}

Square and rectangular plates clamped and simply-supported on a whole edge and subjected by $N_{x y}$ in-plane forces are considered. The intensity of $N_{x y}$ is constant. For the square plate, the boundary and domain discretizations are the same as in Example 1. The results of calculation are presented in Table 3. The first buckling modes are shown in Figure 5. 
For the rectangular plate $\left(l_{x}=2 \cdot l_{y}\right)$ the number of boundary elements is equal to 120 and the number of internal square sub-domains is equal to 200 . The results of the calculation are presented in Table 4 . The first buckling modes for both plates are shown in Figure 6.

Table 3

\section{Critical forces}

\begin{tabular}{|c|c|c|}
\hline \multirow{2}{*}{$\tilde{N}_{\text {cr }}=\tilde{N}_{x y}$} & \multicolumn{2}{|c|}{ Plate } \\
\cline { 2 - 3 } & Clamped & Simply-supported \\
\hline BEM I & 146.833 & 93.009 \\
\hline BEM II & 146.841 & 93.051 \\
\hline Analytical [10] & 145.182 & 92.182 \\
\hline
\end{tabular}

a)

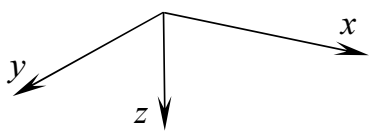

b)

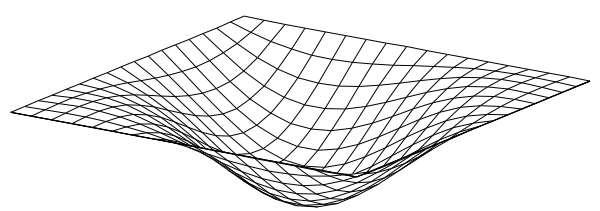

Fig. 5. The first buckling mode for the square plates: a) clamped and b) simply-supported

Table 4

Critical forces

\begin{tabular}{|c|c|c|}
\hline \multirow{2}{*}{$\tilde{N}_{\text {cr }}=\tilde{N}_{x y}$} & \multicolumn{2}{|c|}{ Plate } \\
\cline { 2 - 3 } & Clamped & Simply-supported \\
\hline BEM I & 208.200 & 131.683 \\
\hline BEM II & 208.201 & 131.682 \\
\hline Analytical [10] & 204.103 & 130.279 \\
\hline
\end{tabular}

a)

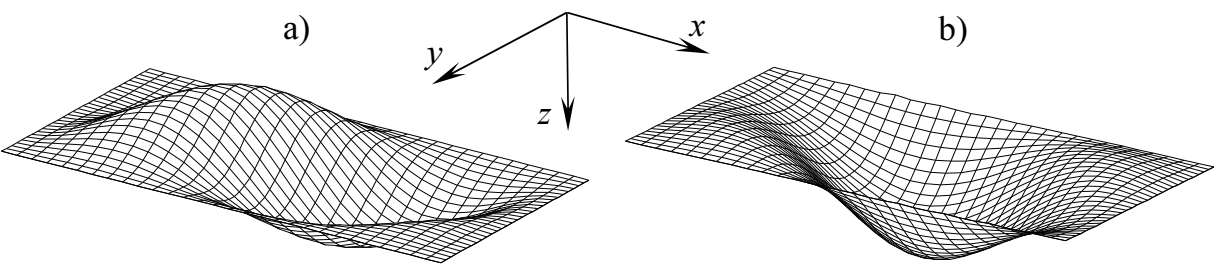

b)

Fig. 6. The first buckling mode of the rectangular plates: a) clamped and b) simply-supported 


\subsection{Example 4}

A square plate, clamped on all edges, is subjected by in-plane compressive forces $N_{x}, N_{y}$ and additionally by tangential forces $N_{x y}$. The intensities of external in-plane forces are constant. The plate boundary and domain discretizations are the same as in Example 1.

The results of calculation are presented in Table 5. The first buckling mode for $N_{x}=N_{y}=N_{x y}$ is shown in the Figure 7a and for $N_{x}=N_{y}=0.5 \cdot N_{x y}$ in the Figure 7b.

Table 5

\section{Critical forces}

\begin{tabular}{|c|c|c|c|c|}
\hline$\tilde{N}_{\text {cr }}$ & $\tilde{N}_{x}=\tilde{N}_{y}=\tilde{N}_{x y}$ & $\tilde{N}_{x}=\tilde{N}_{y}=1.5 \cdot \tilde{N}_{x y}$ & $\tilde{N}_{x}=\tilde{N}_{y}=2 \cdot \tilde{N}_{x y}$ & $\tilde{N}_{x}=\tilde{N}_{y}=0.5 \cdot \tilde{N}_{x y}$ \\
\hline BEM I & 46.305 & 32.964 & 25.395 & 73.990 \\
\hline BEM II & 46.306 & 32.965 & 25.395 & 73.993 \\
\hline Analytical [10] & 44.413 & 31.978 & 24.773 & 69.975 \\
\hline
\end{tabular}

a)

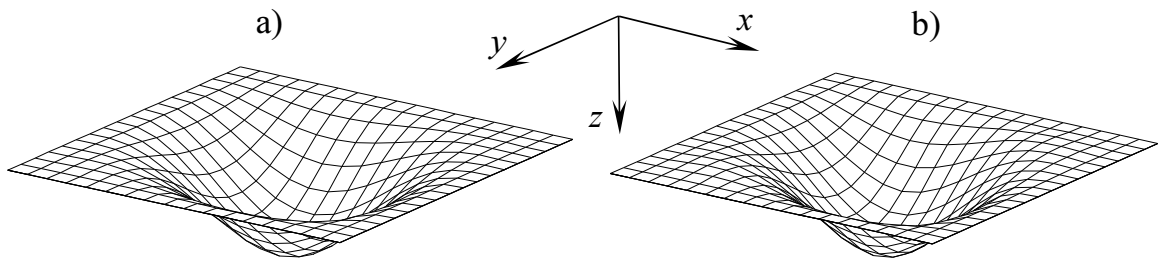

Fig. 7. The first buckling mode of the square clamped plate for: a) $N_{x}=N_{y}=N_{x y}$, b) $N_{x}=N_{y}=0.5 \cdot N_{x y}$

\subsection{Example 5}

A square plate simply-supported on two opposite edges with two edges clamped and subjected by $N_{x y}$ in-plane forces is considered (Fig. 8).

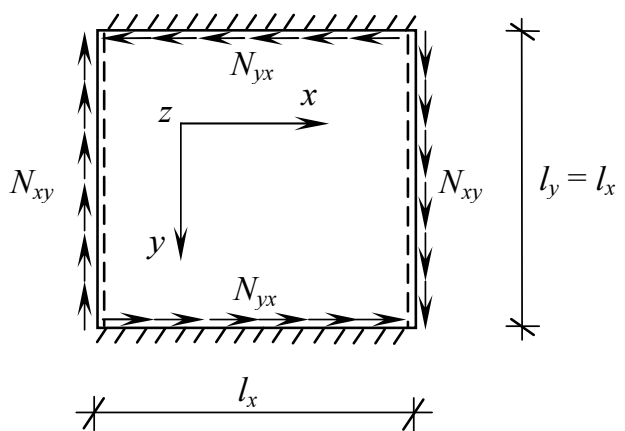

Fig. 8. A square plate simply supported on two opposite edges with two edges clamped 
The intensity of $N_{x y}$ forces is constant. Two types of discretization were adopted:

a) the number of boundary elements is equal to 64 and the number of internal square sub-domains is equal to 256 ;

b) the number of boundary elements is equal to 120 and the number of internal square sub-domains is equal to 400 .

The results of calculation are presented in Table 6 . The first buckling mode is shown in Figure 9.

Table 6

\section{Critical forces}

\begin{tabular}{|c|c|c|c|c|}
\hline \multicolumn{5}{|c|}{$\tilde{N}_{\text {cr }}=\tilde{N}_{x y}$} \\
\hline BEM I(a) & BEM II(a) & BEM I(b) & BEM II(b) & Analytical [10] \\
\hline 125.811 & 125.814 & 125.143 & 125.143 & 122.312 \\
\hline
\end{tabular}

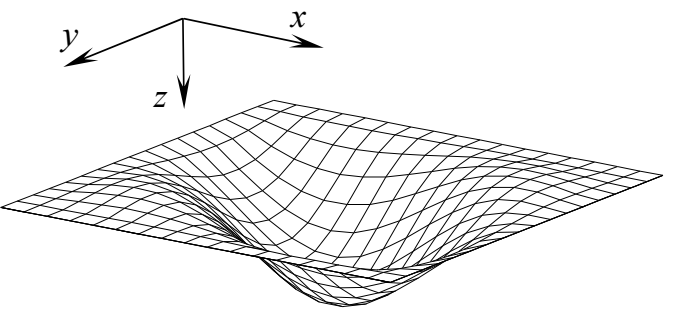

Fig. 9. The first buckling mode of the square plate, simply-supported on two opposite edges with two edges clamped

\subsection{Example 6}

A square and rectangular plates simply-supported and clamped on two opposite edges with two edges free and subjected by $N_{x}$ in-plane forces are considered (Fig. 10).

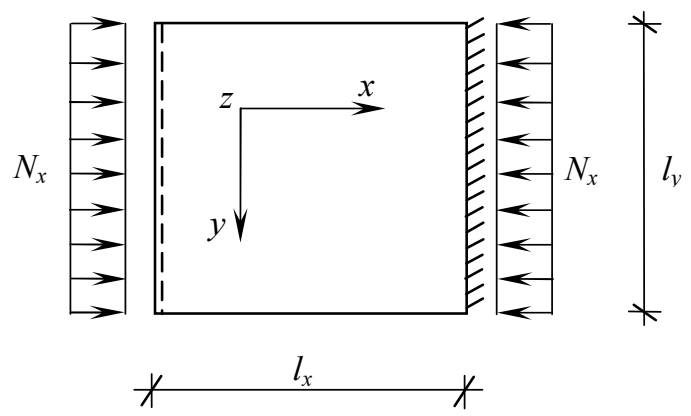

Fig. 10. A square plate simply-supported and clamped on two opposite edges with two edges free 
The intensity of $N_{x}$ forces is constant. For the square plate, the boundary and domain discretizations are the same as in Example 5. The results of calculation are presented in Table 7. The first buckling mode is shown in Figure 11.

Table 7

Critical forces

\begin{tabular}{|c|c|c|}
\hline \multicolumn{3}{|c|}{$\tilde{N}_{\text {cr }}=\tilde{N}_{x}$} \\
\hline BEM II(a) & BEM II(b) & Analytical [10] \\
\hline 19.740 & 19.724 & 18.864 \\
\hline
\end{tabular}

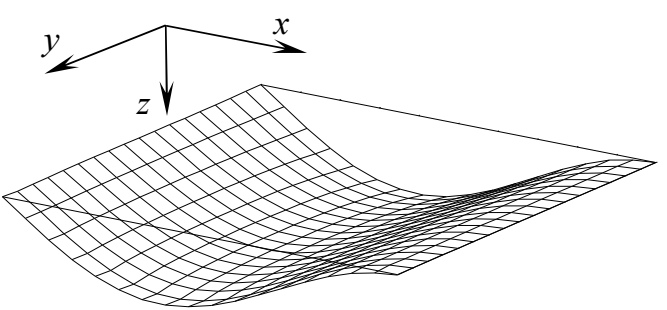

Fig. 11. The first buckling mode of the square plate simply-supported and clamped on two opposite edges with two edges free

The rectangular plate has following relations between its dimensions: $l_{x}=3 \cdot l_{y}$. The number of boundary elements is equal to 120 and the number of internal square sub-domains is equal to 300 . The results of calculation are presented in Table 8. The first buckling mode is shown in Figure 12.

Table 8

\section{Critical forces}

\begin{tabular}{|c|c|}
\hline \multicolumn{2}{|c|}{$\tilde{N}_{\text {cr }}=\tilde{N}_{x}$} \\
\hline BEM II & 6.418 \\
\hline Analytical (beam analogy) & 6.110 \\
\hline
\end{tabular}

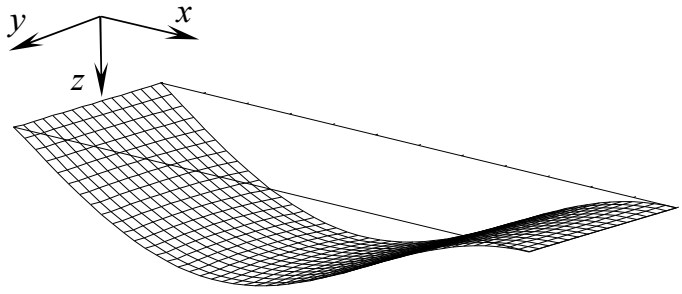

Fig. 12. The first buckling mode of the rectangular plate simply-supported and clamped on two opposite edges with two edges free 


\section{Concluding remarks}

An initial stability of thin plates considering the various conservative load conditions was solved by the Boundary Element Method. This problem was formulated according to the modified approach, in which the boundary conditions are defined so that there is no need to introduce equivalent boundary quantities dictated by the boundary value problem for the biharmonic differential equation. The collocation version of the BEM with singular and non-singular calculations of integrals were employed and the constant type of the boundary element is introduced. The Bèzine technique [2] was used to establish the vector of generalized curvatures inside a plate domain which was divided into rectangular sub-surfaces. The high number of boundary elements and internal sub-domains is not required to obtain sufficient accuracy. The loaded plate edge must be supported in case of external in-plane conservative loading. This condition is required in proposed formulation of buckling analysis. The boundary element results obtained for presented conception of a thin plate bending issue were compared with corresponding analytical solutions derived from classic thin plate [10] and beam theories. The BEM results demonstrate the sufficient effectiveness and efficiency of the proposed approach.

\section{References}

[1] Shi G., Flexural vibration and buckling analysis of orthotropic plates by the Boundary Element Method, International Journal of Solids and Structures 1990, 12, 26, 1351-1370.

[2] Bèzine G., A boundary integral equation method for plate flexure with condition inside the domain, International Journal of Numerical Method in Engineering 1981, 15, 1647-1657.

[3] Nerantzaki M.S., Katsikadelis J.T., Buckling of plates with variable thickness - An Analog Equation Solution, Engineering Analysis with Boundary Elements 1996, 18, 2, 149-154.

[4] Katsikadelis J.T., The analog equation method. A powerful BEM-based solution technique for solving linear and nonlinear engineering problems, [in:] Boundary Element Method XVI, ed. C.A. Brebbia, Computational Mechanics Publications, Southampton 1994, 167-182.

[5] Chinnaboon B., Chucheepsakul S., Katsikadelis J.T., A BEM - based meshless method for buckling analysis of elastic plates with various boundary conditions, International Journal of Structural Stability and Dynamics 2007, 7, 1, 81-89.

[6] Katsikadelis J.T., Babouskos N.G., Nonlinear flutter instability of thin damped plates: A solution by the analog equation method, Journal of Mechanics of Materials and Structures 2009, 4, 7-8, 1395-1414.

[7] Guminiak M., Sygulski R., Initial stability of Kirchhoff plates by the boundary element method using a modified formulation of a boundary condition, Foundations of Civil and Environmental Engineering 2006, 7, 171-186.

[8] Guminiak M., An alternative approach of initial stability analysis of Kirchhoff plates by the Boundary Element Method, Engineering Transactions 2014, 62, 1, 33-59.

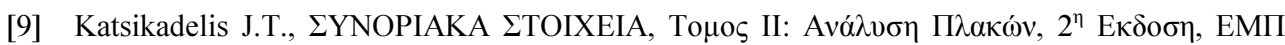
2010 (Boundary Elements, Vol. II, Analysis of Plates, Second Edition, NTUA, Athens 2010 ).

[10] Timoshenko S.P., Gere J.M., Theory of Elastic Stability, Arkady, Warszawa 1962. 